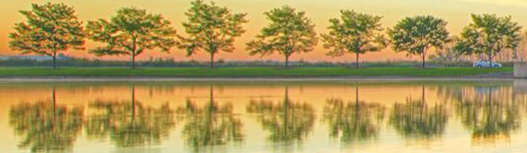

\section{Implementing the Yubikey at Fermilab}

Saúl González and Al Lilianstrom

National Laboratories Information Technology Summit 2019

This manuscript has been authored by Fermi Research Alliance, LLC under Contract No. DE-AC02-07CH11359 with the U.S. Department of Energy, Office of Science, Office of High Energy Physics. 


\section{Implementing the Yubikey at Fermilab}

Fermilab, America's particle physics and accelerator laboratory, is an open science facility. Fermilab started limited use of DOE issued PIV-I cards for elevated access to services as part of the 2016 DOE mandate. With FIPS 140-2 validated Yubikeys now available Fermilab has begun a much broader implementation using the Yubikey as a PIV-I Smart Card, not only to replace the DOE issued cards, but to expand the usage to more users and services as well as network access.

This talk will cover Yubikey provisioning and lifecycle management, authentication service configuration, integration with existing applications and account lifecycle processes, and usage across the unique Fermilab infrastructure.

Track - Infrastructure/Operations 


\section{About Fermilab}

Fermilab is America's particle physics and accelerator laboratory.

- Our vision is to solve the mysteries of matter, energy, space and time for the benefit of all. We strive to:

- lead the world in neutrino science with particle accelerators

- lead the nation in the development of particle colliders and their use for scientific discovery

- advance particle physics through measurements of the cosmos

Our mission is to drive discovery by:

- building and operating world-leading accelerator and detector facilities

- performing pioneering research with national and global partners

- developing new technologies for science that support U.S. industrial competitiveness

www.fnal.gov 


\section{The Fermilab Environment}

- Fermilab is an Open Science Laboratory

- Fermilab's 1,750 employees include scientists and engineers from all around the world.

- Currently hosting over 4000 users

- Fermilab collaborates with more than 50 countries on physics experiments based in the United States and elsewhere. 


\section{Timeline}

- PIV Rollout - September 2016

- Focus on "privileged access"

- Authentication admins

- Network admins

- PII

- Small distribution

- HID ActiveKey evaluation - Summer 2017

- Undertaken by the Authentication Services Group

- Goal was to find an ActiveClient compatible smartcard for use with our Certificate Authority

- Yubikey evaluation - Fall 2017

- Yubikey rollout - Spring 2019 


\section{Authentication Overview}

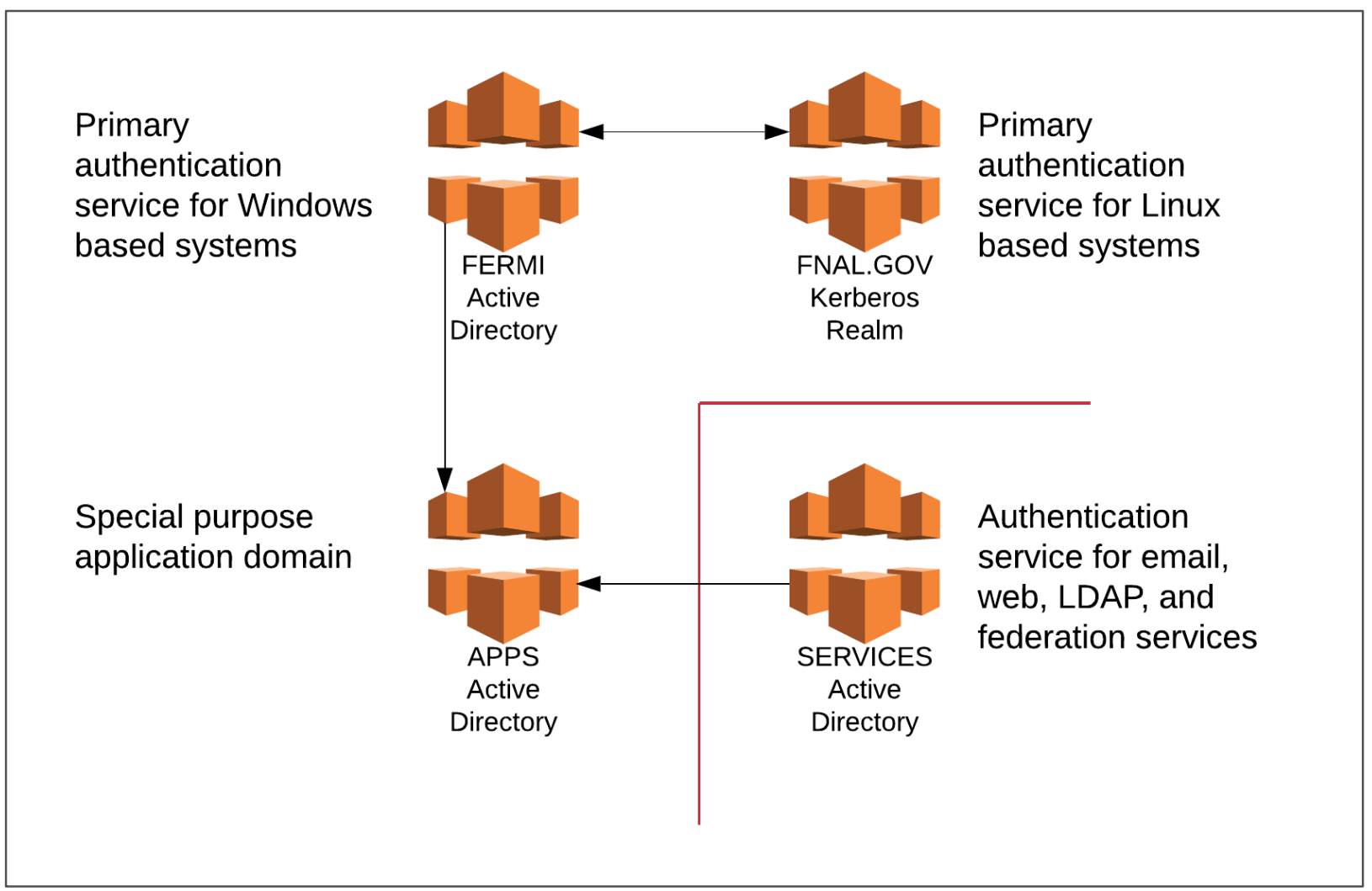




\section{Infrastructure}

- Certificate Authority Servers

- Microsoft Certificate Services integrated with Active Directory

- Offline Root CA

- Subordinate CAs for each Active Directory domain

- User and computer certificates are issued from the subordinate CA

- Certificates for the Root and Subordinate should be published to Active Directory to deploy to all domain members

- Non-domain computers will have to add them

- BYOD

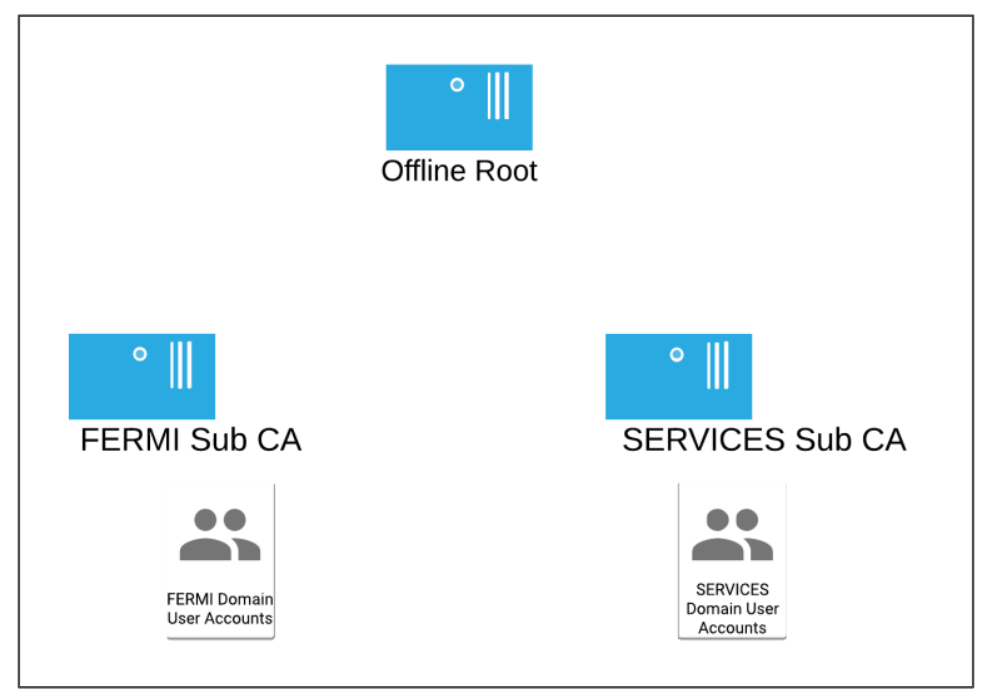

- Non-Windows

- Managed and Stand-alone 


\section{Infrastructure}

- Certificate Revocation List

- CRLs are hosted on a central web server

- CRLs are updated hourly on the subordinate CAs and copied to the web server with a PowerShell script

- Offline Root CA CRL is published and copied to the central web server as part of the monthly patching process

- Exception was required to site policy as a CRL must be located on a plain HTTP site

- Certificates had to be reissued as the CRL evolved

- Individual CA server

- Central web server 


\section{Infrastructure}

庴 File Action View Favorites Window Help

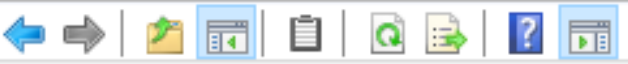

Console Root

圈 Certificates (Local Computer)

$\checkmark$ Personal

Certificates

$>$ Trusted Root Certification Authorities

$>$ Enterprise Trust

$>$ Intermediate Certification Authorities

, 9 Trusted Publichers

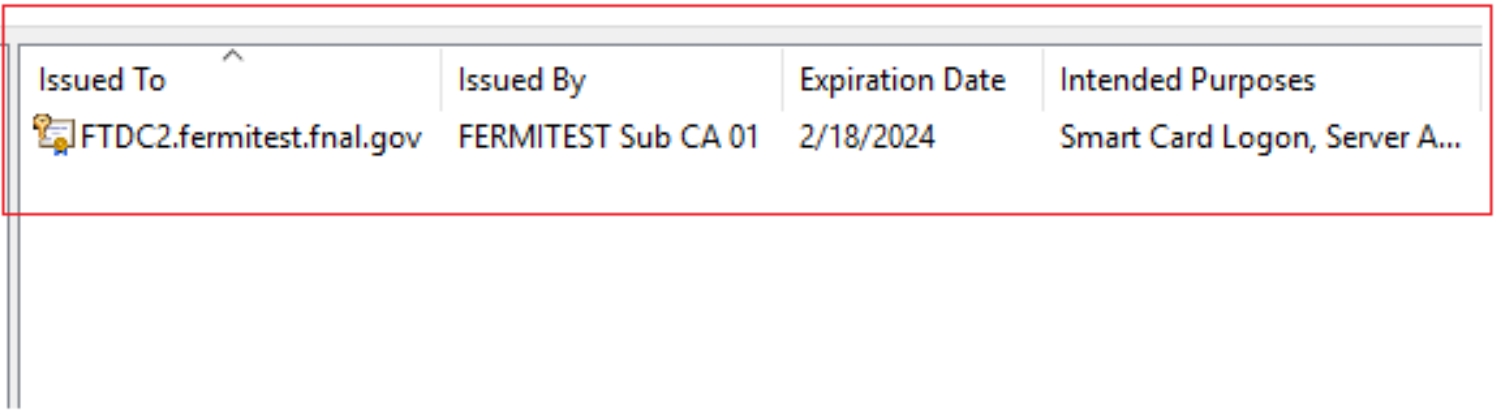

File Action View Favorites Window Help

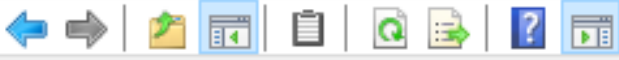

Console Root

圆 Certificates (Local Computer)

畻 Certificates - Service (Active Directory Domain Services)

$\checkmark$ NTDS $\backslash$ Personal

[Certificates

$>$ NTDS $\backslash$ Trusted Root Certification Authorities

$>$ NTDS Enterprise Trust

$>$ NTDS Intermediate Certification Authorities

an nirnm $T$, in i...

\begin{tabular}{|c|c|c|c|}
\hline Issued To & Issued By & Expiration Date & Intended Purposes \\
\hline 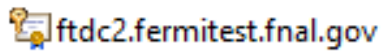 & DigiCert SHA2 High ... & $2 / 3 / 2020$ & Server Authentication, Client Aut \\
\hline
\end{tabular}




\section{Yubikey}

- Database

- Yubikey serial number

- PUK

- Management key

- Username

- Management data

- Identity proofing

- HR business process

- Employees and on-premise contractors only at this time 


\section{Work Flow}

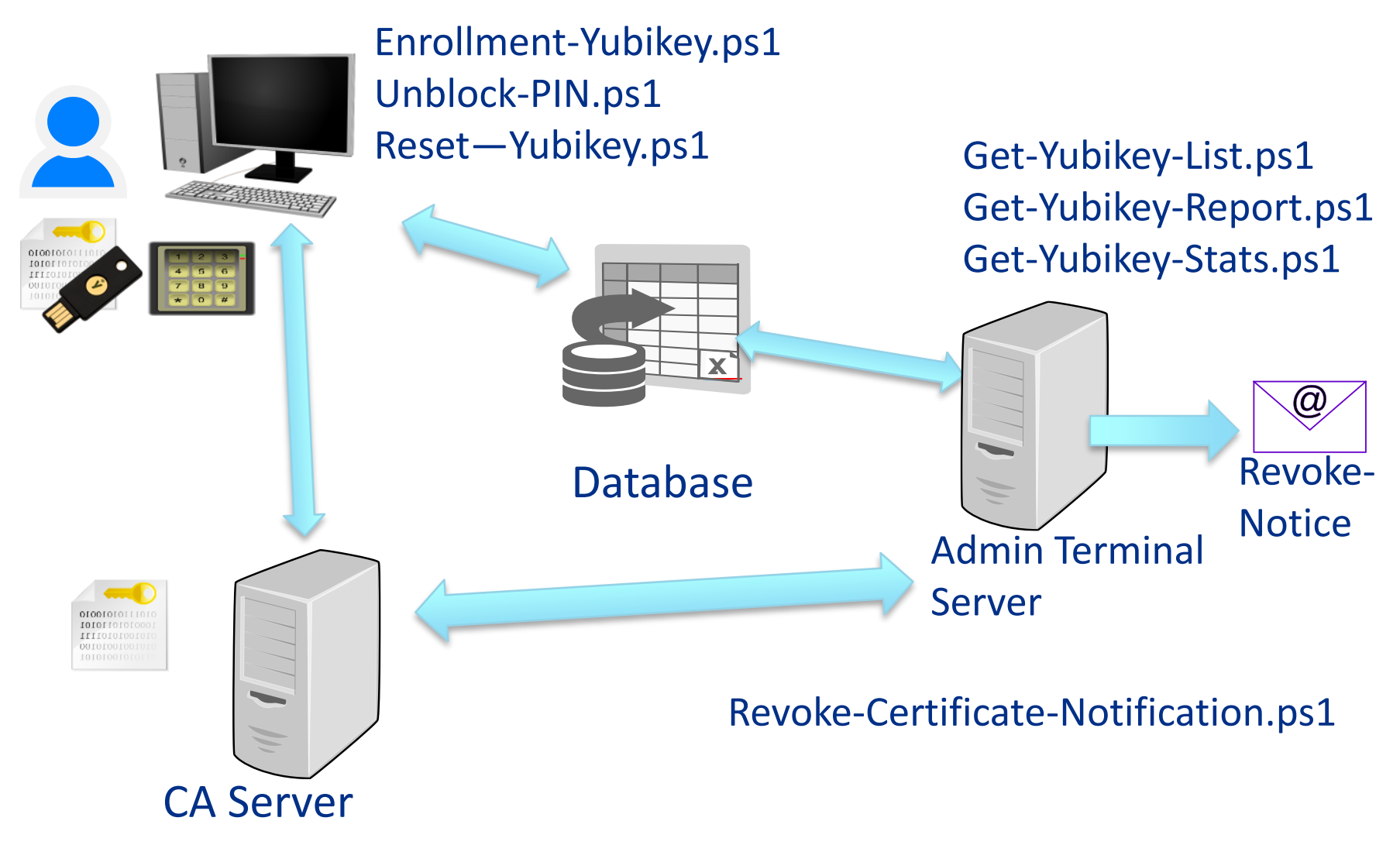


Service Desk Agent:

Keep your assigned Yubikey plugged in.....

During the enrollment process Yubikey devices will be checked....

Removal of Yubikey device could cause application to fail.

[!!! - Insert the User Yubikey

Press Enter to continue:

Identifying Yubikey devices

!!!! - Write down the user"

!!!! - The User's Yubikey Devi

Yubico Yubikey 4 OTP+U2F+CCID

Press ENTER to continue:

Retries have been set to 5

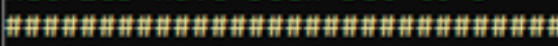

Verify User's Badge ID

Press (C) to Continue or (Q)

Enter User's Badge ID: $632413 \mathrm{~N}$

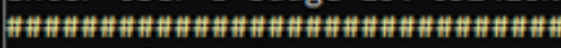

User Information

First Name $=$ Sol

Last Name $=$ Gonz

Logon Name $=$ sgon

Badge $\mathrm{TD}=632413 \mathrm{~N}$

Is the user information correct: Confirm $(\mathrm{Y})$ or $(\mathrm{N})$ - Quit (Q)?

$$
\text { PIN }
$$

Windows Security

\section{Smart Card}

Please enter your PIN.

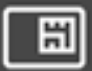

Click here for more information

OK

Cancel 


\section{Yubikey}

- Other functions

- Replacement

- Lost or damaged

- Clearing

- Termination

- Pin/PUK reset

- Yubikey locks after 5 bad P

- With the Replacement or Cle previously issued certificate is Revocation Pending

- PowerShell script queries th

- Contacts CA and revokes

- Updates database owner-auth-adm-auto@listserv.fnal.gov on behalf of FNAL-PKI@fnal.gov

PRIMARY: Certificate To Be Revoked as 04-24-2019 14:41

(i) This message was sent with High importance.

The following certificates need to be revoked.

Thank you

Account Information:

Logon Name: cshepp

Certificate Serial Number: 580000015e06f775583779e9ac00010000015e

Execute C:\Tools \fermi\yubikey \\Revoke-Certificate.ps1 


\section{Uses}

- Application servers

- Windows

- Linux

- Appliances 


\section{Application Servers}

- Windows

- Install Yubikey minidriver

- Diffe Computer Configuration (Enabled)

\begin{tabular}{|c|c|c|c|}
\hline Computer Confi & \multicolumn{2}{|l|}{ Policies } & \\
\hline Policies & \multicolumn{2}{|l|}{ Windows Settings } & hide \\
\hline Administrati & \multicolumn{2}{|l|}{ Security Settings } & hide \\
\hline Poli & \multicolumn{2}{|l|}{ Local Policies/Security Options } & hide \\
\hline Window & \multicolumn{2}{|l|}{ Interactive Logon } & hide \\
\hline \multirow{3}{*}{$\frac{\mathbf{P o}}{\text { Allc }}$} & Policy & Setting & \\
\hline & Interactive logon: Require smart card & Enabled & \\
\hline & Interactive logon: Smart card removal behavior & Lock Workstation & \\
\hline
\end{tabular}




\section{Application Servers}

- Clients for Windows Servers

- Windows Remote Desktop

- Microsoft Remote Desktop for OSX

- rdesktop (OSX and Linux)

- OSX - homebrew (https://brew.sh/)

- brew install homebrew/x11/rdesktop --with-smartcard 


\section{Application Servers}

-

(ㄷ) Start Smart Card Service Properti General Triggers Actions Cond

Name: $\quad$ Start Smart Card Ser

Location:

Author:

Description:

Security options

When running the task, use the $f$ SYSTEM

Run only when user is logged

(2) Run whether user is logged or

$\square$ Do not store password. T

$\square$ Run with highest privileges

$\square$ Hidden

Configure for: (b) Start Smart Card Service Prop General Triggers Actions

When you create a task, you o

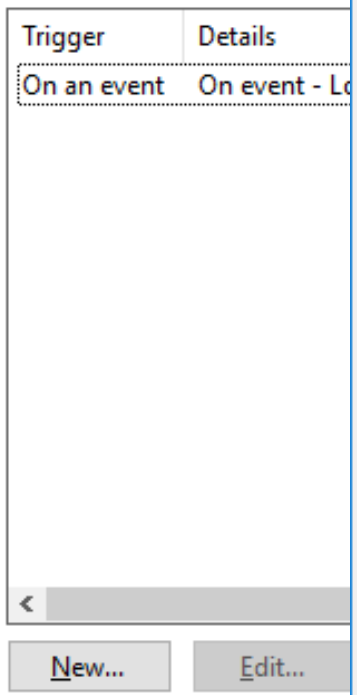

(L) Start Smart Card Service Properties (Local Computer)

General Triggers Actions Conditions Settings History

When you create a task, you must specify the action that will occur when your task starts.

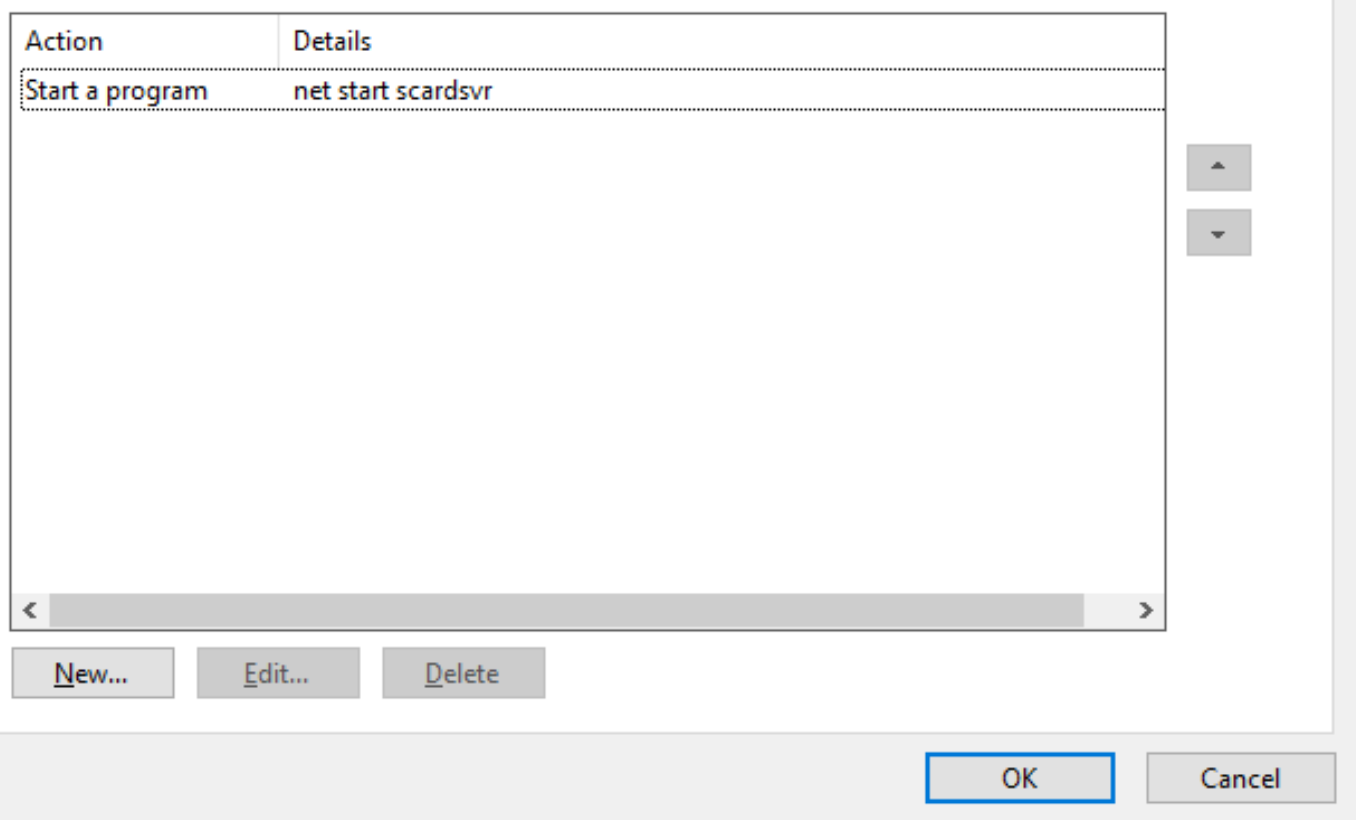




\section{Application Servers}

- Windows

- Certificate subject must be added to the altSecurityldentities attribute for the user in Active Directory

- Default access limited to Domain Admins

1. Delegate access to the Service Desk personnel that issue the Yubikeys and have the issuing script update the attribute

2. Delegate access to a service account that monitors the database and updates the attribute

3. Monitor the database with a script and generate commands to be executed by the domain admins

- Currently - option \#3 is in use

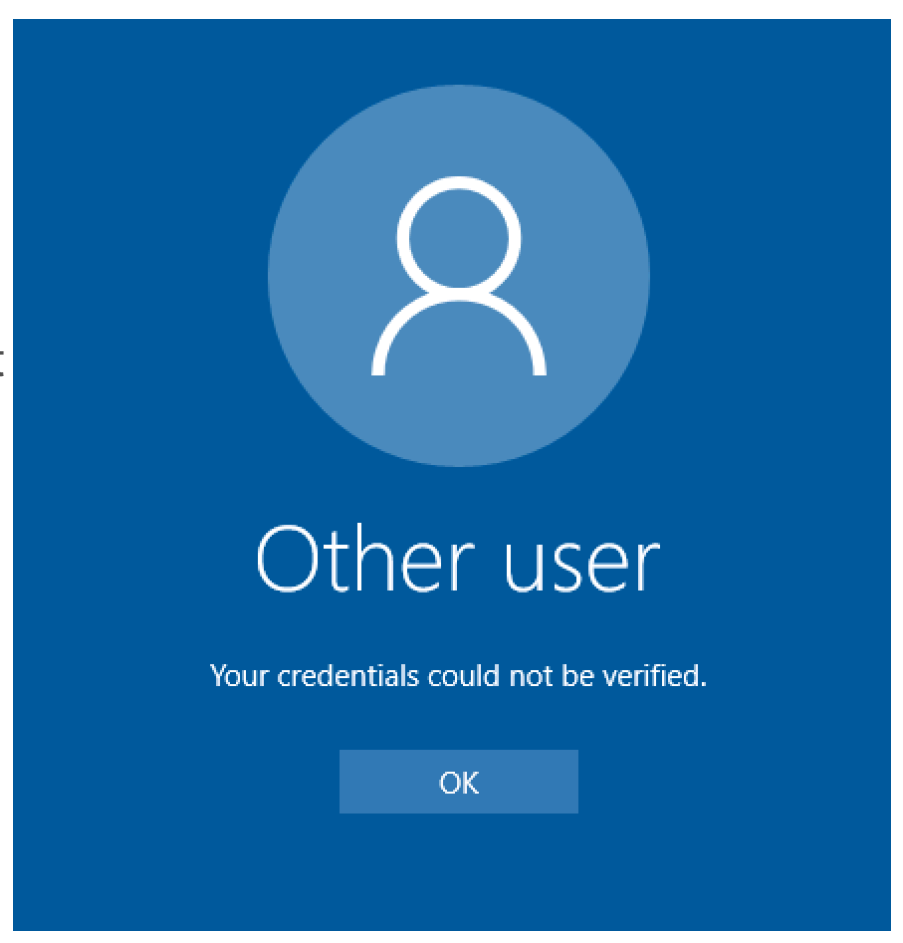




\section{Application Servers}

- Linux

昜 kdc-jump-1.FNAL.GOV - PuTTY

- Configure sshd

- /etc/ssh/sshd_con

- Disable Kerbero

- Enable PubkeyA

- Coordinate with Cyb

- Compliance scann

- Exception was req

- User configuration

- Extract public key

- Add to .ssh/auth

- Be sure to set $p$

- PUTTY Users - a

- PUTTY-CAC

Windows Security

Smart Card

Please enter your PIN.

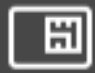

PIN

Click here for more information

OK

Cancel 


\section{Appliances}

- VPN

- MultiFactor authentication

- Citrix

- Replace and extend the existing PIV usage 


\section{Remote locations}

- Deep Underground Neutrino Experiment (DUNE)

- Lead, South Dakota

- Provisioning

- Videoconference assistance for issuing Yubikeys

- Certificate on Yubikey will be on Hold in the CA so it can not be used

- Remote user must contact the Service Desk once the Yubikey arrives to get the default PIN

- Videoconference

- Windows users will be able to reset PIN with Ctrl-Alt-Delete - OSX and Linux...

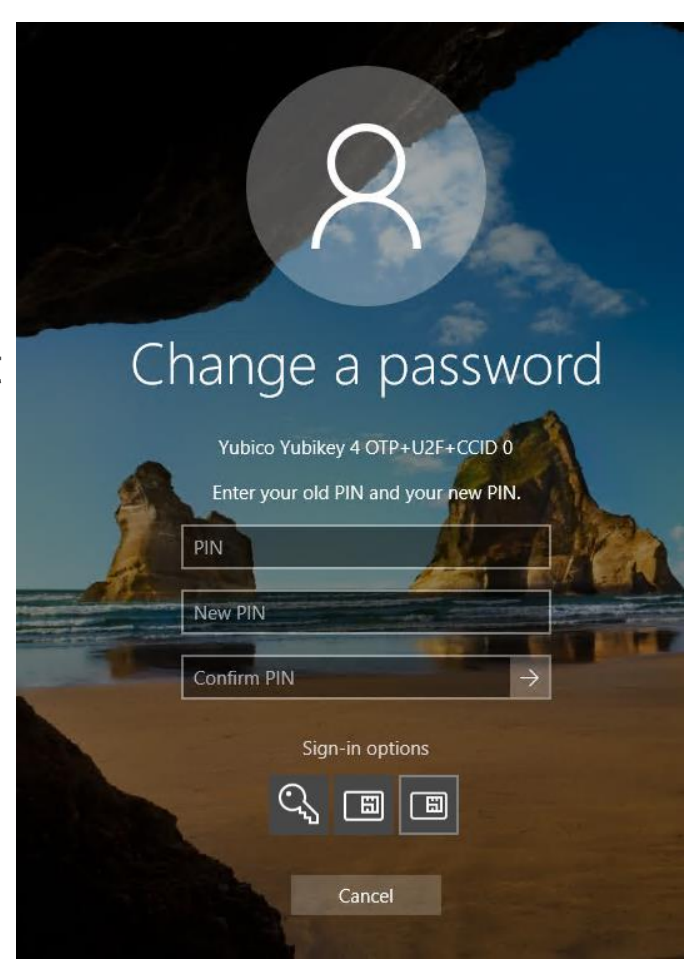




\section{Remote locations}

- User assistance

- PIN Reset

- Locked Yubikey 


\section{Monitoring}

- altSecurityldentities attribute

- Certificates can be added to any account allowing access

- Quest Change Auditor

- Alerts domain administrators whenever the attribute is modified

- PowerShell script

- Checks the certificate assigned against the username of the account

- Notifies domain administrators of any discrepancies
Quest Change Auditor Alert

Monitoring AltSecurityldentity Attribute

Custom AD Object Monitoring: altSecurityldentities attribute was changed for user

win.fnal.gov/General/mithls

Name Value

Coordinator Domain \Name:FENTS1

Agent Domain \Name: OSCAR

Date/Time Detected: 4/22/2019 1:45:19 PM -05:00 - (UTC-06:00) Central Time (US \& Canada)

Date/Time Received: 4/22/2019 1:45:29 PM -05:00 - (UTC-06:00) Central Time (US \& Canada)

\begin{tabular}{|c|c|}
\hline Source: & Change Auditor \\
\hline User: & FER \sgon-a-da \\
\hline \multicolumn{2}{|l|}{ Initiator: } \\
\hline Origin Server: & OSCAR \\
\hline Origin IPv4: & 23.75 .345 .200 \\
\hline Agent: & OSCAR \\
\hline Action: & Add Attribute \\
\hline \multicolumn{2}{|l|}{ From: } \\
\hline To: & X509: $\langle 1\rangle \mathrm{DC}=\mathrm{gov}, \mathrm{DC}=$ fnal, $\mathrm{DC}=$ win, $\mathrm{CN}=\mathrm{FERMI}$ Sub CA $01<\mathrm{S}\rangle \mathrm{CN}=$ mithls \\
\hline Result: & Success \\
\hline
\end{tabular}




\section{Metrics}

- PowerShell script to query the dat

- In the past week

- Since issuance started

- PowerShell script to create graphs

- Get-Corpchart PowerShell script

- https://me.ahasayen.com/

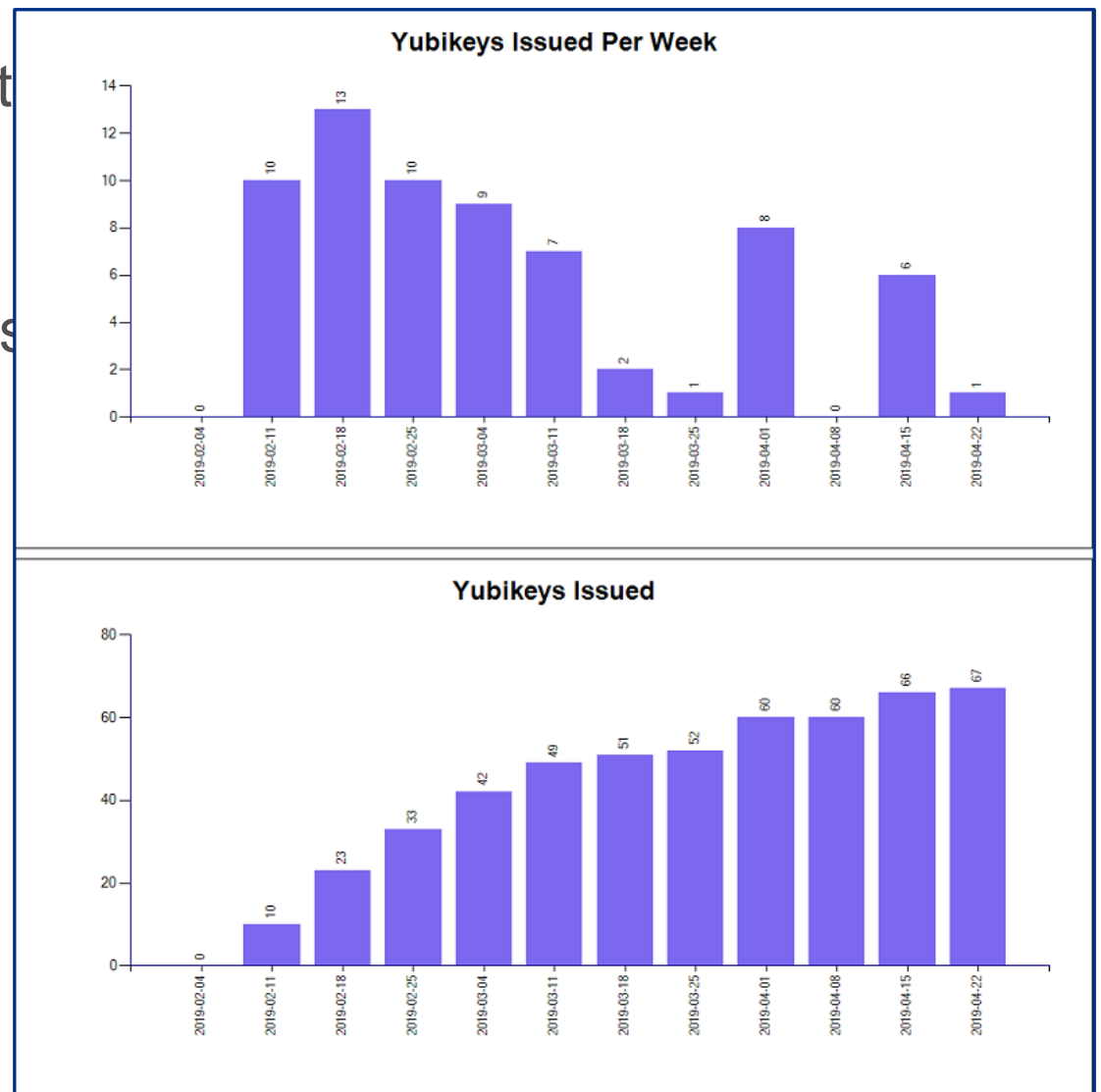




\section{Future plans}

- Desktop logon

- Get rid of passwords

- SSO Integration

- Step up for certain URLS

- MFA for select sites

- FNAL.GOV Kerberos realm integration 


\section{Lessons learned}

- Use of the PIV is still required for access of the One ID web service

- Scheduled tasks running under service accounts on SmartCard required servers

- If you need to start a command prompt as the service account your certificate needs to be added to the service accounts altSecurityldentities attribute

- This allows runas /smartcard /user:domainlserviceaccount $\mathbf{c m d}$ to work

- Potential security issue

- Monitor changes to altSecurityldentities

- A revoked certificate on a Yubikey can still be used for Public Key SSH

- No check of the CRL

- Appliances do not check the Delta CRLs produced by the Microsoft CA server 


\section{Questions}

